\title{
Modeling and Nonlinear Response of the Cam-Follower Oblique-Impact System
}

\author{
Yong-Feng Yang, ${ }^{1}$ Yu Lu, ${ }^{1}$ Ting-Dong Jiang, ${ }^{1,2}$ and $\mathrm{Na} \mathrm{Lu}{ }^{1}$ \\ ${ }^{1}$ Institute of Vibration Engineering, Northwestern Polytechnical University, Xi'an 710072, China \\ ${ }^{2}$ Guizhou Aero-Engine Research Institute, Guiyang 550081, China \\ Correspondence should be addressed to Yong-Feng Yang; yyf@nwpu.edu.cn
}

Received 4 May 2016; Accepted 20 June 2016

Academic Editor: Massimiliano Ferrara

Copyright (c) 2016 Yong-Feng Yang et al. This is an open access article distributed under the Creative Commons Attribution License, which permits unrestricted use, distribution, and reproduction in any medium, provided the original work is properly cited.

In order to quickly and accurately analyze the complex behavior of cam-follower oblique-impact system, a mathematical model which can describe separation, impact, and contact was established in this paper. The transient impact hypothesis was extended, and the oblique collision model was established by considering the tangential slip. Moreau time-stepping method was employed to solve the linear complementarity problem which transformed by the oblique-impact equations. The simulation results show that the cam and follower kept permanent contact when the cam rotational speed was low. With the increase of the cam rotational speed, the cam and follower would be separated and then impact under the gravity action. The system performance shows very complex nonlinear characteristics.

\section{Introduction}

Cam-follower devices are important classical impacting systems which are used in a wide range of applications. The rotation of the cam at some constant speed provides the force to operate the follower. The most common example is valve trains of internal combustion engines, where the cam rotation imparts through the follower the proper motion to the engine valves while a spring provides the restoring force necessary to maintain contact between the components [1]. The impact of cam-follower is a typical oblique impact. In analyses using nonsmooth dynamics, oblique impact of rough bodies in an unsymmetrical configuration can result in self-locking or "jam" at the sliding contact if the coefficient of friction is sufficiently large: this has been termed Painleve's paradox [2]. Because of this, the follower may exhibit very complex behavior including bifurcations and chaos.

Alzate et al. [3] show that a sudden transition to chaos is observed in an experimental cam-follower. In their mathematical model, the tangential friction effect is ignored. Considering the effect of tangential friction, Sundar et al. [4] study the linear response of a cam-follower. But they do not discuss the nonlinear characteristic. Some researches studied the beam's analysis and experimental methods that are similar and can be used in our study. For example, Ding et al. $[5,6]$ investigates the convergence of the Galerkin method for the dynamic response of an elastic beam resting on a nonlinear foundation with viscous damping subject to a moving concentrated load. Zhang et al. [7] present experimental verifications of vibration suppression for a cantilever beam bond with a piezoelectric actuator by an adaptive controller. In our research, the transient impact hypothesis is extended, and the oblique collision model is established by considering the tangential slip. Moreau time-stepping method is employed to solve the linear complementarity problem which transforms by the oblique-impact equations. The complex behavior including bifurcations and chaos is shown by the simulation.

\section{Linear Complementarity Problem}

A linear complementarity problem (LCP) is a problem of the form $\mathbf{y}=\mathbf{M x}+\mathbf{b}, \mathbf{y} \geq 0, \mathbf{x} \geq 0$, and $\mathbf{y}^{T} \mathbf{x}=0$, where $\mathbf{M}$ is an $n \times n$ matrix and $\mathbf{x}, \mathbf{y}$, and $\mathbf{b}$ are an $n$-dimensional vector [8].

LCP equations may have unique solution, no solution, or multiple solutions. Many problems in scientific computing and engineering applications may lead to solutions of LCP. 


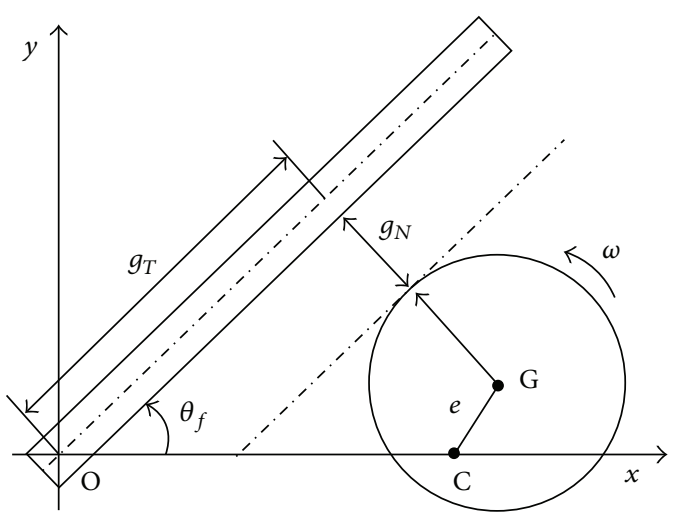

FIGURE 1: Sketch of cam-follower oblique-impact model.

For example, the LCP may arise from application problems such as the linear and quadratic programming, the economies with institutional restrictions upon prices, the optimal stopping in Markov chain, and the free boundary problems. Several computational methods have been developed for solving LCPs. The two basic functions of the LCP are Upr function and Sgn function:

$$
\begin{gathered}
\mathrm{Upr}= \begin{cases}\{0\} & x>0 \\
(-\infty, 0] & x=0 \\
\varnothing & x<0,\end{cases} \\
\operatorname{Sgn}=\partial|x|= \begin{cases}\{1\} & x>0 \\
{[-1,1]} & x=0 \\
\{-1\} & x<0 .\end{cases}
\end{gathered}
$$

In mechanics, Upr function is used to simulate geometry and dynamics of unilateral constraint, and Sgn function is used to simulate various kinds of dry friction.

\section{Oblique-Impact Model}

A cam-follower with oblique-impact on the contact point is studied in Figure 1. The summary of the general notations used in Figure 1 is shown in Notations. By extending the transient impact hypothesis and considering the tangential slip, the oblique collision model is established.

In the present work, the normal contact between rigid bodies is characterized by a set-valued force law called Signorini's condition [9]. Figure 2 shows two convex rigid bodies apart from each other by a relative normal gap or distance denoted by $g_{N}$. The relative normal gap is nonnegative due to bodies' impenetrability condition, being the two bodies in contact with each other when $g_{N}=0$. According to Signorini's condition, the normal contact conditions are

$$
\begin{array}{ll}
g_{N}=0 \wedge \lambda_{N} \geq 0 & (\text { closed contact }), \\
g_{N}>0 \wedge \lambda_{N}=0 & \text { (open contact) } .
\end{array}
$$

Equation (2) represents a complementarity behavior; always zero is the product of the relative normal gap and normal contact force; that is, $g_{N} \lambda_{N}=0$.

The classical Coulomb friction law is another typical example that can be considered as a set-valued force law [9]. For the two conditions sliding and sticking, the tangential forces are

$$
\begin{aligned}
& -\lambda_{T}=\mu \lambda_{N} \operatorname{Sgn}\left(\gamma_{T}\right), \quad \gamma_{T} \neq 0 \text { (sliding), } \\
& -\mu \lambda_{N} \leq \lambda_{T} \leq \mu \lambda_{N} \quad \text { (sticking), }
\end{aligned}
$$

where $\mu$ is the friction coefficient; $\lambda_{N}$ is the normal contact force; and $\gamma_{T}$ is the relative tangential velocity. These two conditions can be summarized by a set-valued force law as [9]

$$
-\lambda_{T} \in \mu \lambda_{N} \operatorname{Sgn}\left(\gamma_{T}\right) .
$$

Tangential Coulomb's contact states can be expressed by Sgn function. It also represents a complementarity behavior.

\section{Dynamics Equations of the Follower}

For the model shown in Figure 1, the dynamics of follower under different speed conditions are considered. There are three states of the follower and cam: separate, contact, and impact. The dynamic equations of the follower can be expressed as [10]

$$
\begin{aligned}
M \dot{u}+h=0 & \text { separate, } \\
M \dot{u}+h-w_{N} \lambda_{N}-w_{T} \lambda_{T}=0 & \text { contact, } \\
M\left(u^{+}-u^{-}\right)-w_{N} \Lambda_{N}-w_{T} \Lambda_{T}=0 & \text { impact, }
\end{aligned}
$$

where $u$ is generalized velocity and here is the follower's angular velocity $\dot{\theta}_{f} ; M=J_{o}=m l^{2} / 3+m r^{2} / 4$ is the follower's moment of inertia; $h=m g l \cos \left(\theta_{f}\right) / 2$ is the follower's external force; $w$ is the generalized coefficient in contact point; $\Lambda$ is the force of the follower, and $u^{+}$and $u^{-}$are the velocity before and after the impact point, respectively. Notice that $\lambda_{N}$ is a continuous boundary parameter when there is no impact. $\lambda_{T}$ is a noncontinuous parameter when there is viscous-to-slip transition or change in the direction of relative speed. $N$ denotes normal direction and $T$ denotes tangential direction.

The relationship between $w_{N}, w_{T}$ and contact point's relative speeds is

$$
\begin{aligned}
& \gamma_{N}=w_{N} u+\frac{\partial w_{N}}{\partial t} \\
& \gamma_{T}=w_{T} u+\frac{\partial w_{T}}{\partial t} .
\end{aligned}
$$

In order to get the unknown parameters $w_{N}$ and $w_{T}$, we need to calculate the distance function in Figure 1:

$$
\begin{aligned}
g_{N}= & {\left[x_{0}+e \cos \left(\theta_{c}\right)\right] \sin \left(\theta_{f}\right)-e \sin \left(\theta_{c}\right) \cos \left(\theta_{f}\right) } \\
& -r-R, \\
g_{T}= & {\left[x_{0}+e \cos \left(\theta_{c}\right)\right] \cos \left(\theta_{f}\right)+e \sin \left(\theta_{c}\right) \sin \left(\theta_{f}\right) . }
\end{aligned}
$$




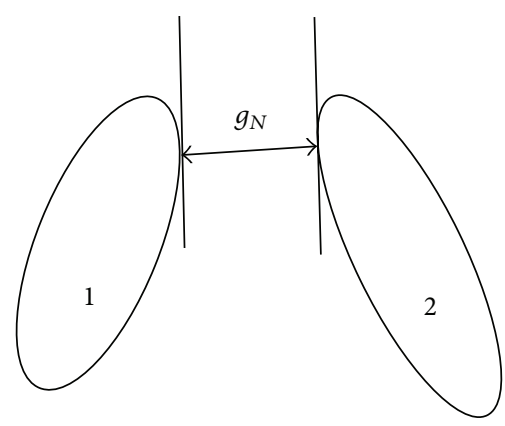

(a)

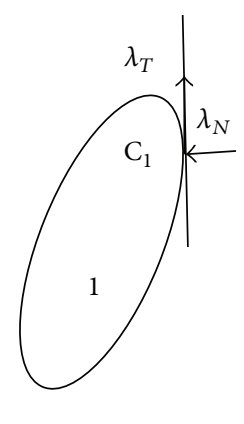

(b)

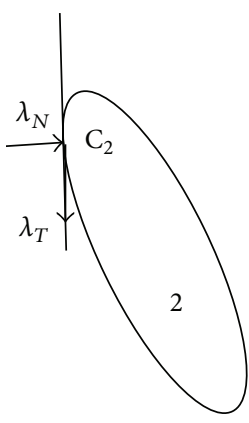

FIGURE 2: Sketch of rigid body contact: (a) normal distance $g_{N}$ and (b) tangential and normal force.

According to the results of [11],

$$
\dot{g}=w \dot{q}+\frac{\partial w}{\partial t} .
$$

Therefore,

$$
\begin{aligned}
w_{N}= & \left(x_{0}+e \cos \left(\theta_{c}\right)\right) \cos \left(\theta_{f}\right)+e \sin \left(\theta_{c}\right) \sin \left(\theta_{f}\right), \\
w_{T}= & -\left(x_{0}+e \cos \left(\theta_{c}\right)\right) \sin \left(\theta_{f}\right) \\
& +e \sin \left(\theta_{c}\right) \cos \left(\theta_{f}\right) .
\end{aligned}
$$

There are three equations for the cam-follower impact system. In order to quickly and accurately analyze the complex behavior of cam-follower oblique-impact system, it is better to establish one mathematical equation which can describe separation, impact, and contact motion. Equation measure is introduced here. Equation (6) is multiplied by the Lebesgue measure $d t$ and (7) is multiplied by the atoms measure $d \eta$. Then add the two equations:

$$
\begin{gathered}
M\left[\dot{u} d t+\left(u^{+}-u^{-}\right) d \eta\right]+h d t-w_{N}\left(\lambda_{N} d t+\Lambda_{N} d \eta\right) \\
-w_{T}\left(\lambda_{T} d t+\Lambda_{T} d \eta\right)=0 .
\end{gathered}
$$

This equation can be abbreviated as

$$
M d u+h d t-w_{N} d P_{N}-w_{T} d P_{T}=0,
$$

where $d u=\dot{u} d t+\left(u^{+}-u^{-}\right) d \eta$ is velocity measure. It contains two parts: one is the continuous part, Lebesgue measure $\dot{u} d t$; the other is the noncontinuous part, Dirac measure. When the cam-follower is in the nonimpact state, the velocity measure $d u=\dot{u} d t$. The impact measure which relates to the Lagrange multiplier also contains two parts: $d P=\lambda d t+\Lambda d \eta$. The separation, impact, and contact equations are expressed as the unified form in (13) [10].

Moreau time-stepping method [10] is employed to discretize (13). We can get

$$
M_{M}\left(u_{E}-u_{A}\right)+h_{M} \Delta t-w_{N M} p_{N}-w_{T M} p_{T}=0,
$$

where $A$ and $E$ represent starting and ending time. $M$ is the middle time of $A$ and $E$. For the normal impact parameter $p_{N}$, apply the Upr function:

$$
-p_{N} \in \operatorname{Upr}\left(\xi_{N}\right) \Longleftrightarrow p_{N} \geq 0, \xi_{N} \geq 0, p_{N} \xi_{N}=0,
$$

where $\xi_{N}=\gamma_{N}^{+}+\varepsilon_{N} \gamma_{N}^{-}$.
In order to get the expectation LCP condition, we need to separate Sgn function to two Upr functions. For the tangential impact parameter $p_{T}$, we can get

$$
\begin{array}{ll} 
& -p_{T} \in \mu p_{N} \operatorname{Sgn}\left(\xi_{T}\right) \Longleftrightarrow \exists \xi_{R}, \xi_{L} \\
\text { s.t. } & \mu p_{N}+p_{T} \geq 0, \xi_{R} \geq 0,\left(\mu p_{N}+p_{T}\right) \xi_{R}=0 \\
& \mu p_{N}-p_{T} \geq 0, \xi_{L} \geq 0,\left(\mu p_{N}-p_{T}\right) \xi_{L}=0 \\
& \xi_{T}=\xi_{R}-\xi_{L} .
\end{array}
$$

For the sake of brevity, we define $p_{R}:=\mu p_{N}+p_{T}$ and $p_{L}:=\mu p_{N}-p_{T}$, where ":=" means "is defined as." The second and third expressions of (16) are $P_{R} \geq 0, \xi_{R} \geq 0, P_{R} \xi_{R}=0$ and $P_{L} \geq 0, \xi_{L} \geq 0, P_{L} \xi_{L}=0$. The LCP condition is

$$
0 \leq\left(\begin{array}{c}
\xi_{N} \\
\xi_{R} \\
p_{L}
\end{array}\right) \perp\left(\begin{array}{c}
p_{N} \\
p_{R} \\
\xi_{L}
\end{array}\right) \geq 0 .
$$

The full LCP equation of follower can be expressed as

$$
\begin{aligned}
& \left(\begin{array}{c}
\xi_{N} \\
\xi_{R} \\
p_{L}
\end{array}\right) \\
& =\left(\begin{array}{ccc}
w_{N M} M_{M}^{-1}\left(w_{N M}-w_{T M} \mu\right) & w_{N M} M_{M}^{-1} w_{T M} & 0 \\
w_{T M} M_{M}^{-1}\left(w_{N M}-w_{T M} \mu\right) & w_{N M} M_{M}^{-1} w_{T M} & 1 \\
2 \mu & -1 & 0
\end{array}\right)\left(\begin{array}{c}
p_{N} \\
p_{R} \\
\xi_{L}
\end{array}\right) \\
& +\left(\begin{array}{c}
w_{N M} M_{M}^{-1} h_{M} \Delta t+\left(1+\varepsilon_{N}\right) \gamma_{N A} \\
w_{T M} M_{M}^{-1} h_{M} \Delta t+\left(1+\varepsilon_{N}\right) \gamma_{T A} \\
0
\end{array}\right) .
\end{aligned}
$$

From the solution of (17) and (18), we can get $p_{N}$ and $p_{R}$. Then the generalized velocity can be calculated by

$$
\begin{aligned}
u_{E}= & M_{M}^{-1}\left(w_{N M}-w_{T M} \mu\right) p_{N}+M_{M}^{-1} w_{T M} p_{R} \\
& +M_{M}^{-1} h_{M} \Delta t+u_{A}
\end{aligned}
$$

and the generalized displacement can be gotten by the definition of Moreau time time-stepping method. 


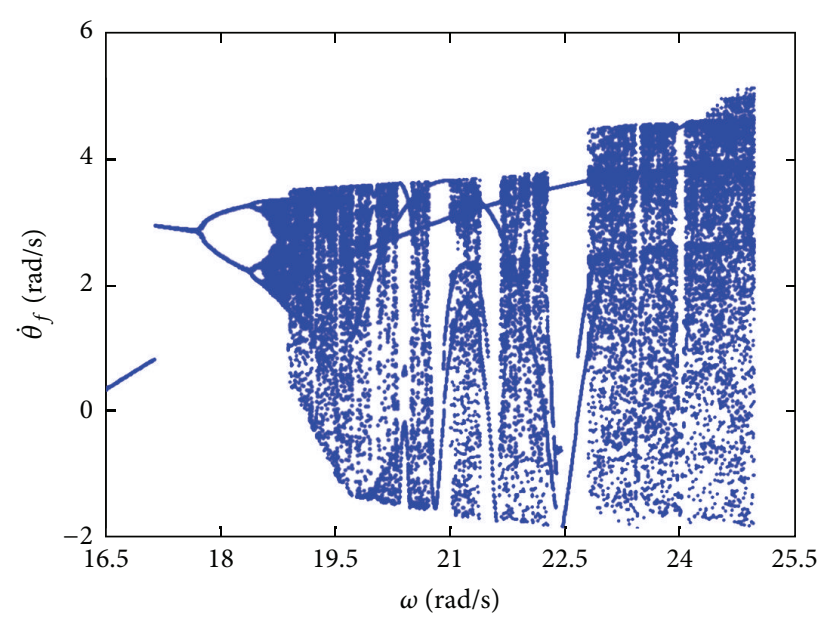

(a)

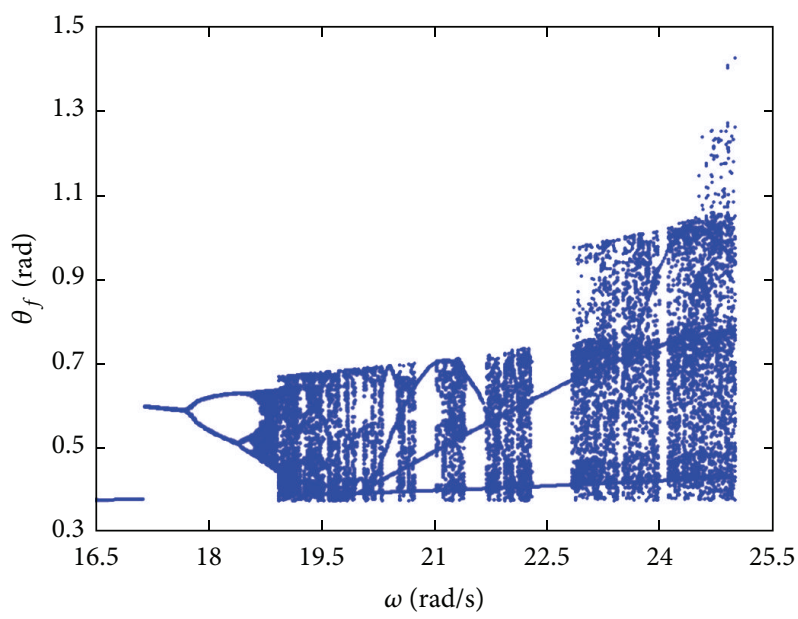

(b)

FIGURE 3: Bifurcation diagram with the change of cam speed: (a) angular velocity and (b) angular displacement.

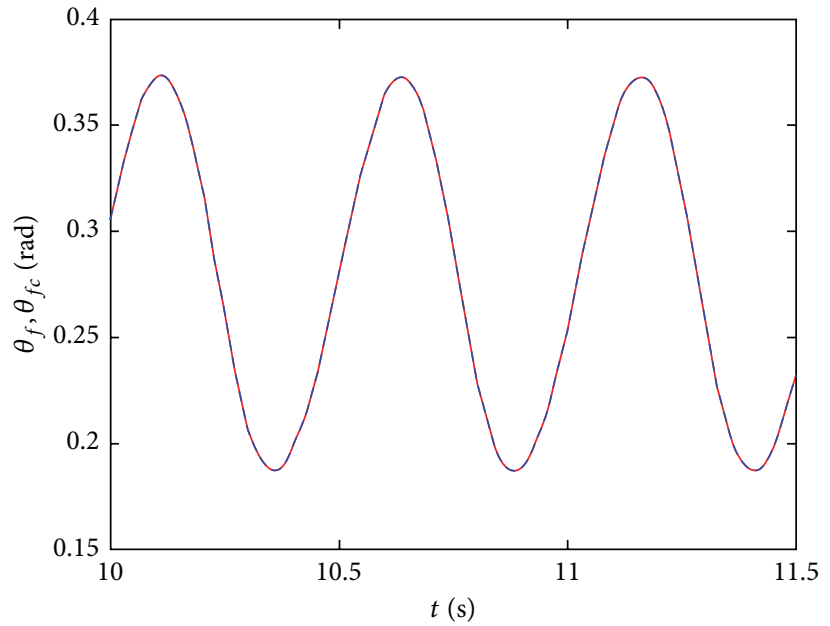

(a)

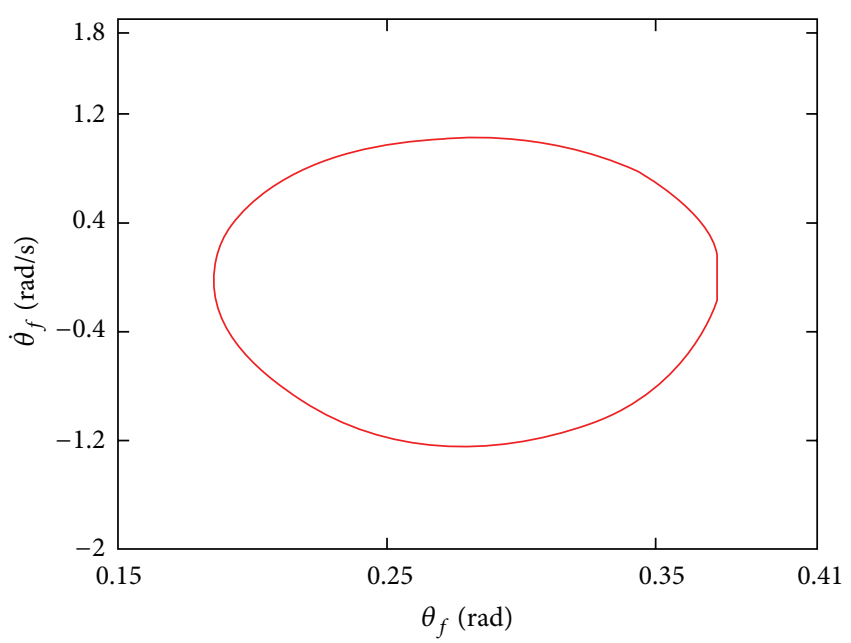

(b)

FIGURE 4: Cam and follower permanent contact when $\omega=12 \mathrm{rad} / \mathrm{s}$ : (a) time history of $\theta_{f}$ (solid line) and $\theta_{f c}$ (dashed line) and (b) phase diagram.

\section{Simulation of the Dynamics Response}

MATLAB is employed for simulation of the equations. In order to get a proper accuracy, the time step of Moreau midpoint method must be very small. We choose $0.0002 \mathrm{~s}$ as the time step, and the values of other parameters can be found in Table 1.

With the increasing of the cam rotational speed, the state of the cam-follower is transformed from the initial permanent contact to the separation and impact. The system performs very complex nonlinear characteristics, such as period, quasiperiod, and chaos response. The bifurcation diagrams are shown in Figure 3.

5.1. Permanent Contact. When the cam rotational speed is low, the constraining force between cam and follower is less than the follower's restoring force. Therefore, the cam and follower keep in contact. The angular displacement of time history diagram and phase diagram is shown in Figure 4 when $\omega=12 \mathrm{rad} / \mathrm{s}$. The motion of cam and follower remained the same (there are a solid line and a dashed line in Figure 4(a)), and the phase plane shows a smooth circle.

5.2. Period Motion. With the increasing of the cam speed, the cam and follower would be separated. When the cam and follower impact, there are minor collisions after the first impact, and then follower would contact the cam's surface until the next separation. As shown in Figure 5, the motion of the follower is periodic 1 or the orbit is $P(\infty, 1)$.

When the cam speed goes higher, as shown in Figures 6 and 7 , the responses are $P(1,1), P(2,2), P(4,4), \ldots .$. From the time history of these $P(n, n)$ responses, the common point is that there is only 1 impact in each cam period. 


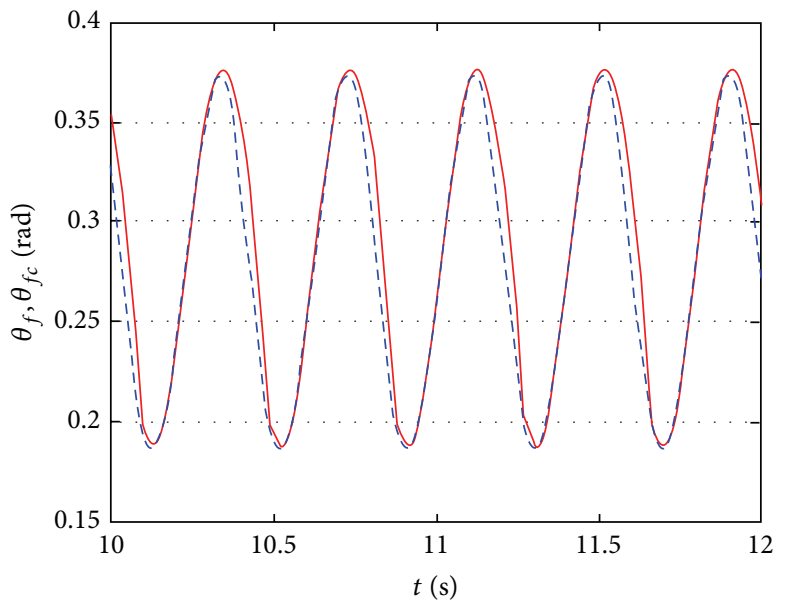

(a)

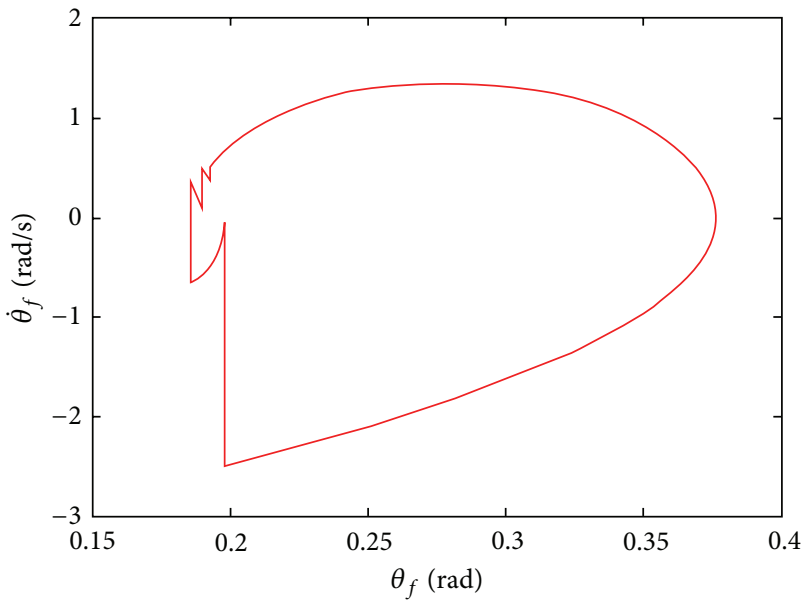

(b)

Figure 5: $P(\infty, 1)$ motion when $\omega=16 \mathrm{rad} / \mathrm{s}$ : (a) time history of $\theta_{f}$ (solid line) and $\theta_{f c}$ (dashed line) and (b) phase diagram.

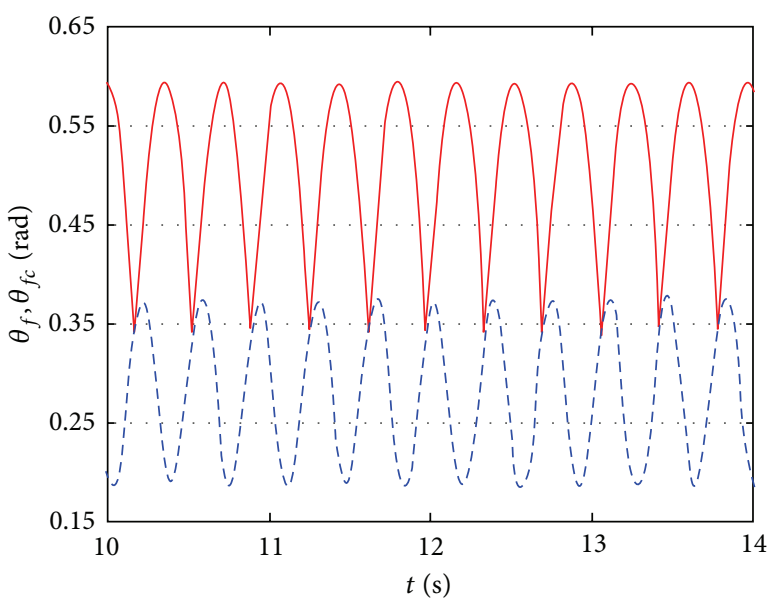

(a)

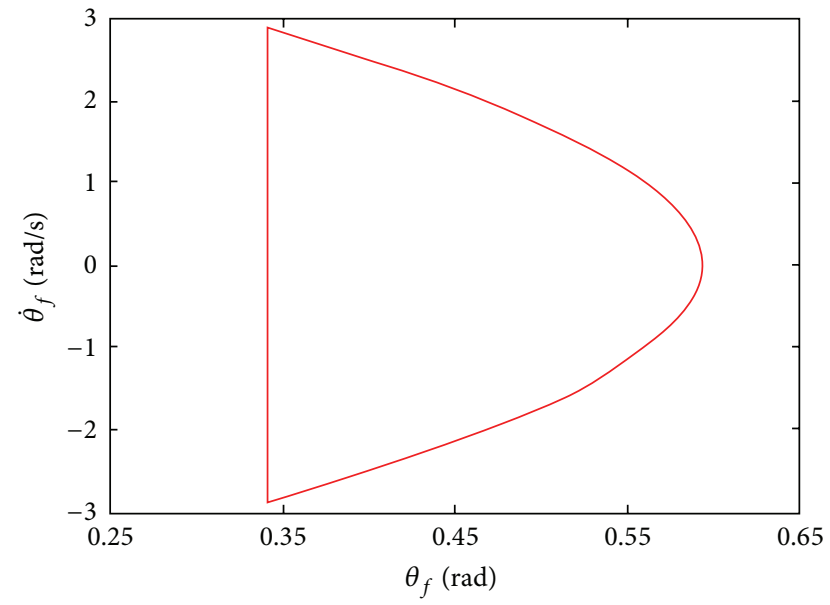

(b)

Figure 6: $P(1,1)$ motion when $\omega=17.4 \mathrm{rad} / \mathrm{s}$ : (a) time history of $\theta_{f}$ (solid line) and $\theta_{f_{c}}$ (dashed line) and (b) phase diagram.

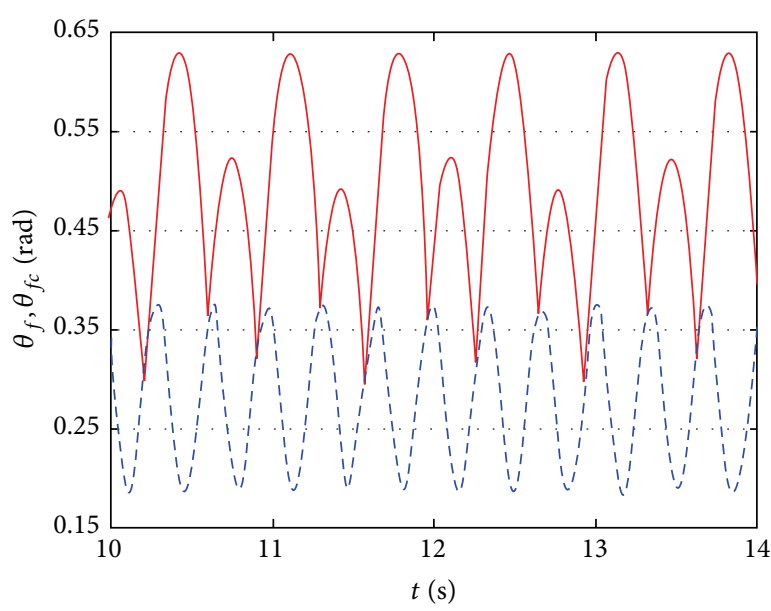

(a)

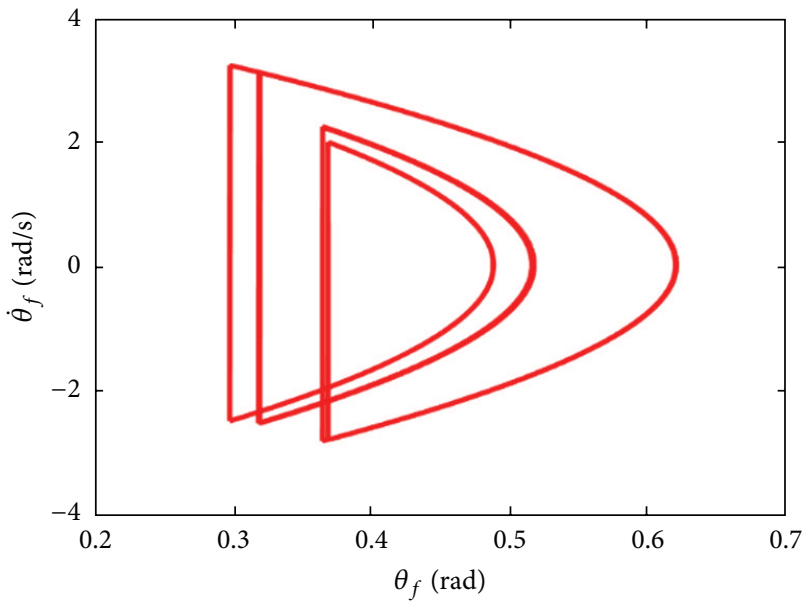

(b)

Figure 7: $P(4,4)$ motion when $\omega=18.5 \mathrm{rad} / \mathrm{s}$ : (a) time history of $\theta_{f}$ (solid line) and $\theta_{f c}$ (dashed line) and (b) phase diagram. 


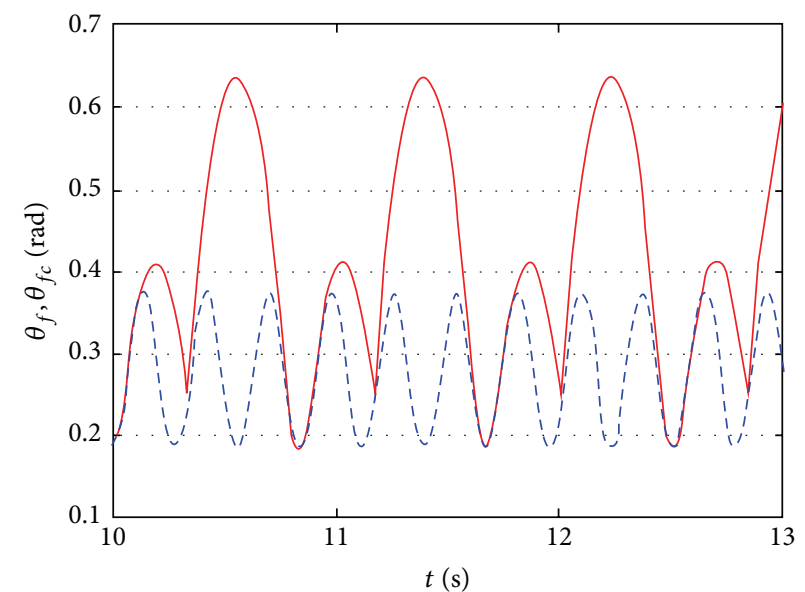

(a)

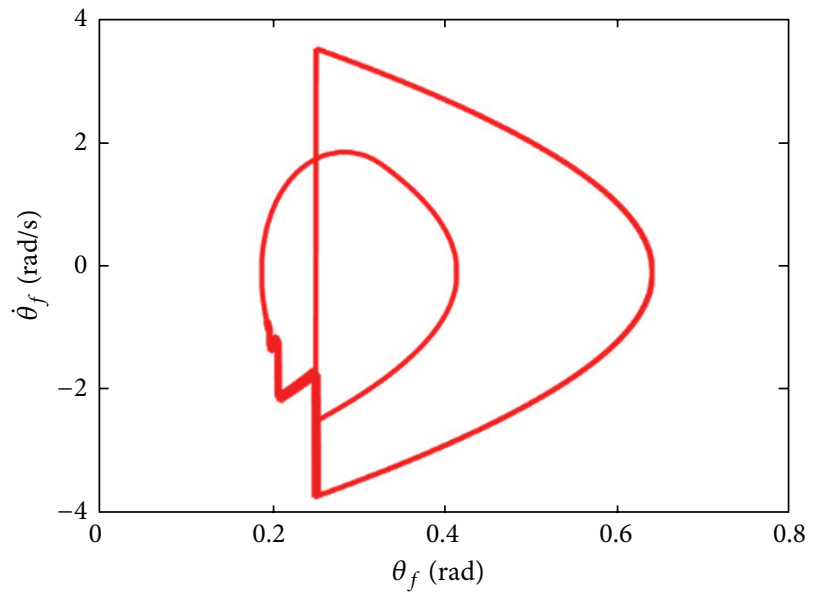

(b)

Figure 8: $P(\infty, 2)$ motion when $\omega=22.5 \mathrm{rad} / \mathrm{s}$ : (a) time history of $\theta_{f}$ (solid line) and $\theta_{f_{c}}$ (dashed line) and (b) phase diagram.

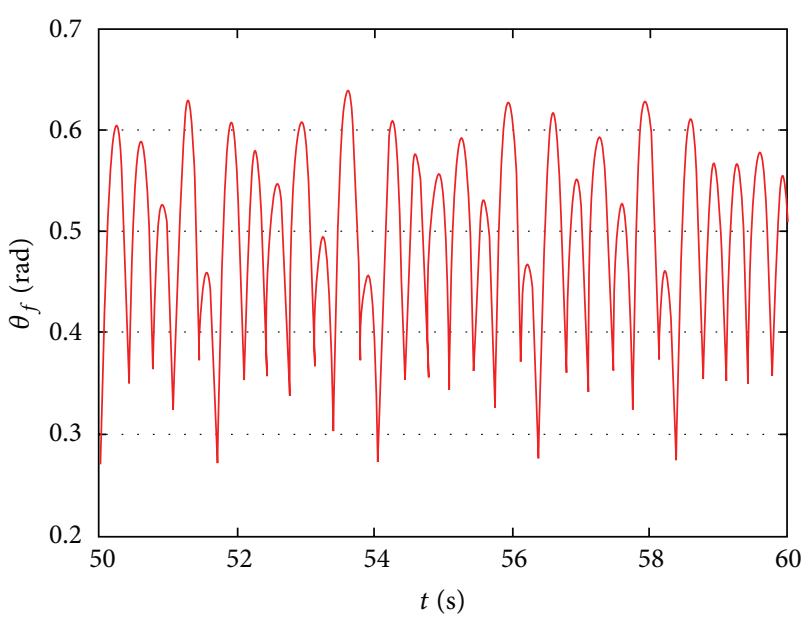

(a)

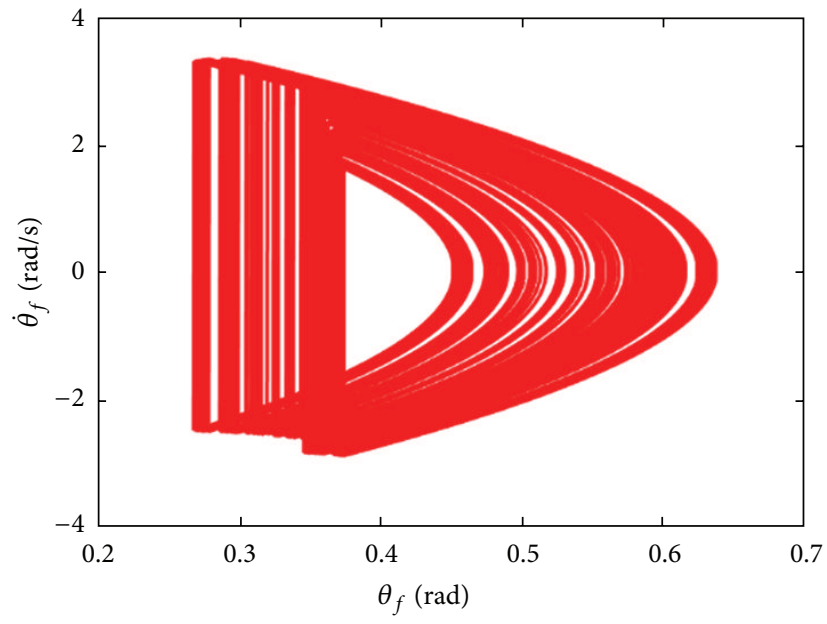

(b)

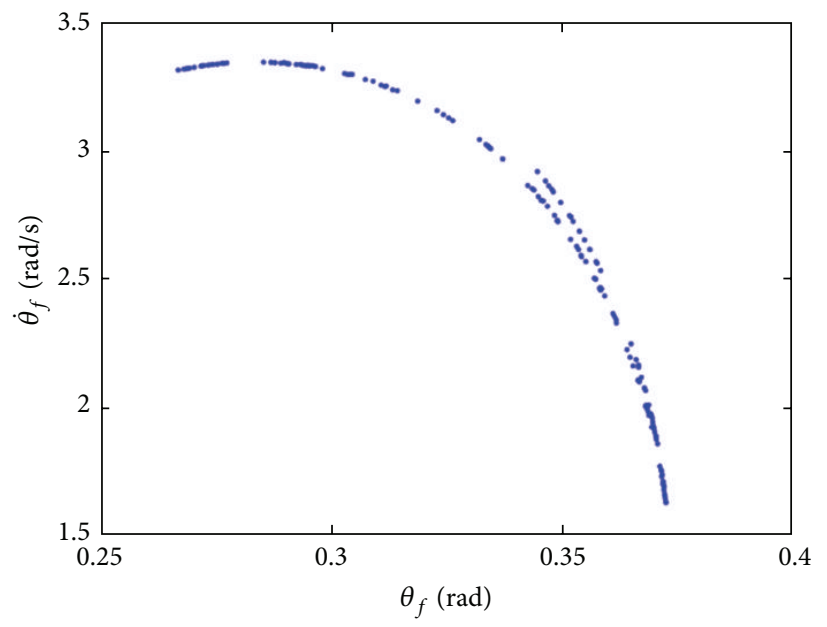

(c)

FIgURE 9: Chaos motion when $\omega=18.84 \mathrm{rad} / \mathrm{s}$ : (a) time history; (b) phase diagram; and (c) Poincaré map. 


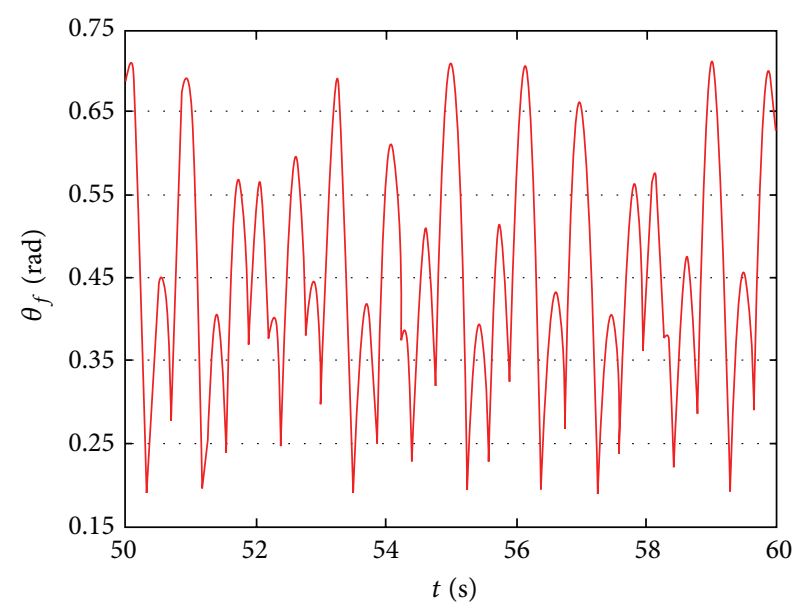

(a)

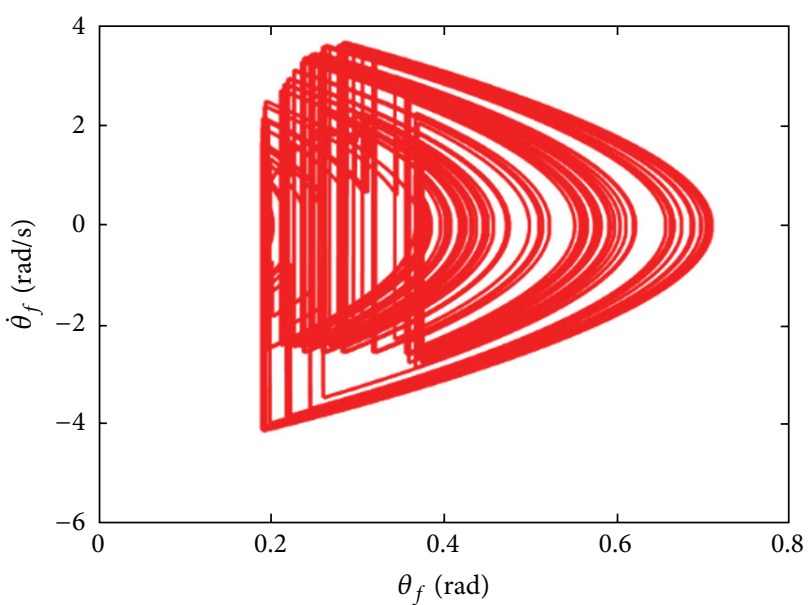

(b)

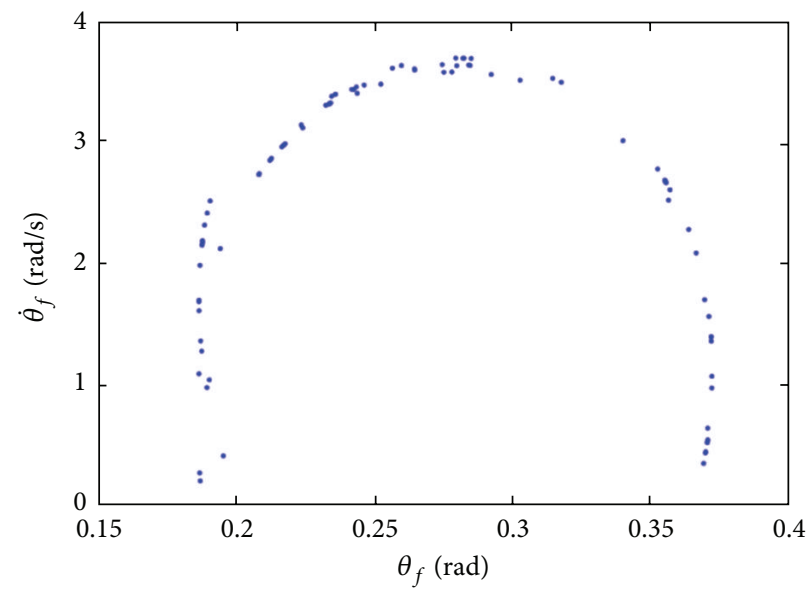

(c)

FIgURE 10: Chaos motion when $\omega=21.7 \mathrm{rad} / \mathrm{s}$ : (a) time history; (b) phase diagram; and (c) Poincaré map.

TABle 1: Cam-follower oblique-impact numerical simulation parameters.

\begin{tabular}{lc}
\hline Parameter & Value \\
\hline Length of the follower, $L$ & $0.8 \mathrm{~m}$ \\
Radius of the follower, $r$ & $0.01 \mathrm{~m}$ \\
Mass of the follower, $m$ & $1.975 \mathrm{~kg}$ \\
Eccentricity of the cam, $e$ & $0.036 \mathrm{~m}$ \\
Radius of the cam, $R$ & $0.1 \mathrm{~m}$ \\
Coefficient of restitution in normal direction & 0.42 \\
Coefficient of restitution in tangential direction & 0.4 \\
Friction coefficient, $\mu$ & 0.2 \\
Distance between bear fix point of the follower and & $0.4 \mathrm{~m}$ \\
rotation center of the cam, $x_{0}$ & $0 \mathrm{rad}$ \\
\hline Initial angular position of the cam, $\theta_{0}$ &
\end{tabular}

There also exist $P(\infty, n)$ motions. The rotation speeds of these motions are between two chaos areas in Figure 3. Figure 8 shows the $P(\infty, 2)$ motion. For these motions, the period of impact is greater than the cam. When the collision occurs, the normal velocity of cam and follower is close. Therefore, collision could not change the follower's angular velocity direction.

5.3. Chaos Motion. Generally, for vibroimpact system, there are two ways for Poincare section selection: (1) to select the fixed phase section of trajectory as Poincaré and (2) to select the collision surface as Poincaré section. In this paper, the collision surface is used:

$$
\Sigma=\left\{(\theta, \dot{\theta}, t) \in R^{2} \times R^{+} \mid \xi_{N}=0, \gamma_{N A}<0\right\}
$$

$\xi_{N}=0$ represents the collision point, and the mirror collision can be excluded by $\gamma_{N A}<0$.

Figure 9 shows the response when $\omega=18.84 \mathrm{rad} / \mathrm{s}$. The collision does not follow the periodic variation and became chaotic. Figure 10 shows the response when $\omega=21.7 \mathrm{rad} / \mathrm{s}$. Compared with Figure 9, the chaos response shows more characteristics of slip phenomenon with $21.7 \mathrm{rad} / \mathrm{s}$. 


\section{Conclusions}

The cam and follower oblique collision model is established in this paper. In order to simulate the response, the equations of the separation, impact, and contact motion are transformed to a linear complementarity problem. The main conclusions are as follows:

(1) The contact and tangential impact law represent a complementarity behavior. LCP equation method could be used to solve the oblique collision problem.

(2) The system performs very complex nonlinear characteristics, such as period, quasiperiod, and chaos response. For every motion, there is a special collision phenomenon.

\section{Notations}

$r: \quad$ Radius of the follower

$O$ : Bear fix point of the follower

$L$ : Length of the follower

$\theta_{f}$ : Angular position of follower with counterclockwise sense of rotation

$\theta_{c}$ : Angular position of cam with counterclockwise sense of rotation

$e: \quad$ Eccentricity of the cam

$\omega$ : Rotation speed of the cam

$C$ : Rotation center of the cam

$G$ : Geometric center of the cam

$\theta_{0}$ : Initial angular position of the cam

$g_{N}$ : Displacement between cam and follower

$g_{T}$ : Displacement between follower's fix point and the point which is nearest to the cam.

\section{Competing Interests}

The authors declare that they have no competing interests.

\section{Acknowledgments}

This work was supported by the National Natural Science Foundation of China (Grants nos. 11272257 and 11672251) and NPU Aoxiang New Star.

\section{References}

[1] R. Alzate, M. Di Bernardo, U. Montanaro, and S. Santini, "Experimental and numerical verification of bifurcations and chaos in cam-follower impacting systems," Nonlinear Dynamics, vol. 50, no. 3, pp. 409-429, 2007.

[2] Y. Shen and W. J. Stronge, "Painleve paradox during oblique impact with friction," European Journal of Mechanics. A. Solids, vol. 30, no. 4, pp. 457-467, 2011.

[3] R. Alzate, P. T. Piiroinen, and M. Di Bernardo, "From complete to incomplete chattering: a novel route to chaos in impacting cam-follower systems," International Journal of Bifurcation and Chaos, vol. 22, no. 5, Article ID 1250102, 2012.

[4] S. Sundar, J. T. Dreyer, and R. Singh, "Rotational sliding contact dynamics in a non-linear cam-follower system as excited by a periodic motion," Journal of Sound and Vibration, vol. 332, no. 18, pp. 4280-4295, 2013.

[5] H. Ding, L.-Q. Chen, and S.-P. Yang, "Convergence of Galerkin truncation for dynamic response of finite beams on nonlinear foundations under a moving load," Journal of Sound and Vibration, vol. 331, no. 10, pp. 2426-2442, 2012.

[6] H. Ding and L.-Q. Chen, "Galerkin methods for natural frequencies of high-speed axially moving beams," Journal of Sound and Vibration, vol. 329, no. 17, pp. 3484-3494, 2010.

[7] T. Zhang, H. G. Li, Z. Y. Zhong, and G. P. Cai, "Hysteresis model and adaptive vibration suppression for a smart beam with time delay," Journal of Sound and Vibration, vol. 358, pp. 35-47, 2015.

[8] M. B. Meingast, M. Legrand, and C. Pierre, "A linear complementarity problem formulation for periodic solutions to unilateral contact problems," International Journal of NonLinear Mechanics, vol. 66, pp. 18-27, 2014.

[9] R. I. Leine and C. Glocker, "A set-valued force law for spatial Coulomb-Contensou friction," European Journal of Mechanics-A/Solids, vol. 22, no. 2, pp. 193-216, 2003.

[10] P. Flores, R. Leine, and C. Glocker, "Application of the nonsmooth dynamics approach to model and analysis of the contactimpact events in cam-follower systems," Nonlinear Dynamics, vol. 69, no. 4, pp. 2117-2133, 2012.

[11] C. Glocker, "On frictionless impact models in rigid-body systems," Philosophical Transactions of the Royal Society of London B: Biological Sciences, vol. 359, no. 1789, pp. 2385-2404, 2001. 


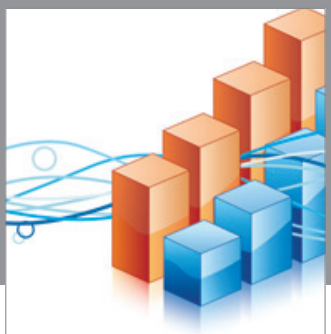

Advances in

Operations Research

vatem alat4

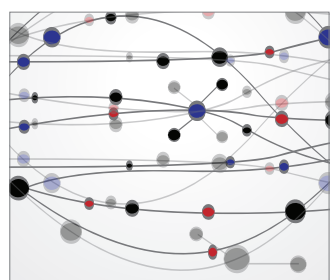

\section{The Scientific} World Journal
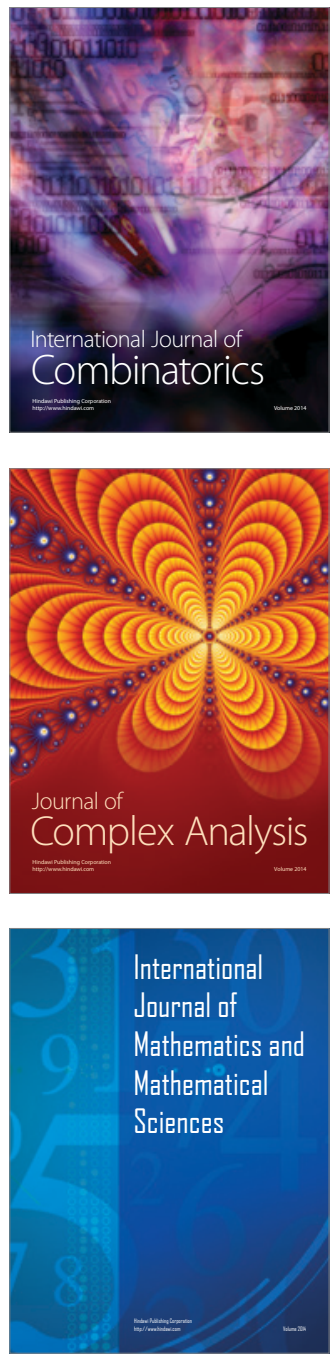
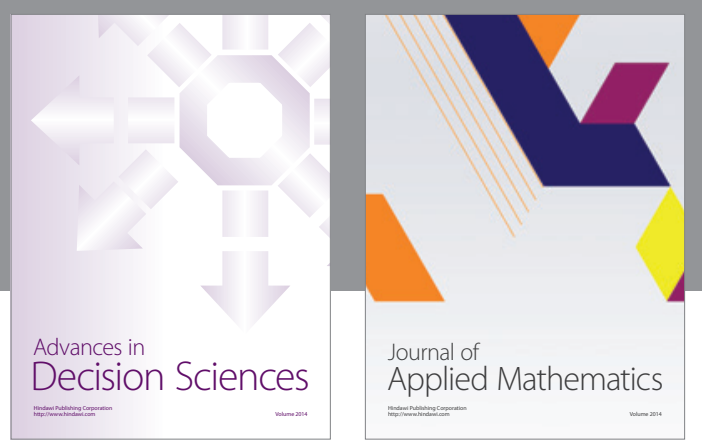

Algebra

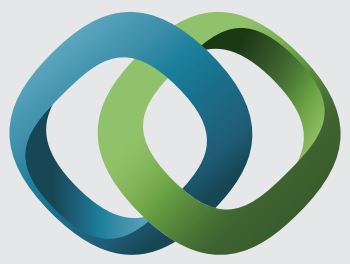

\section{Hindawi}

Submit your manuscripts at

http://www.hindawi.com
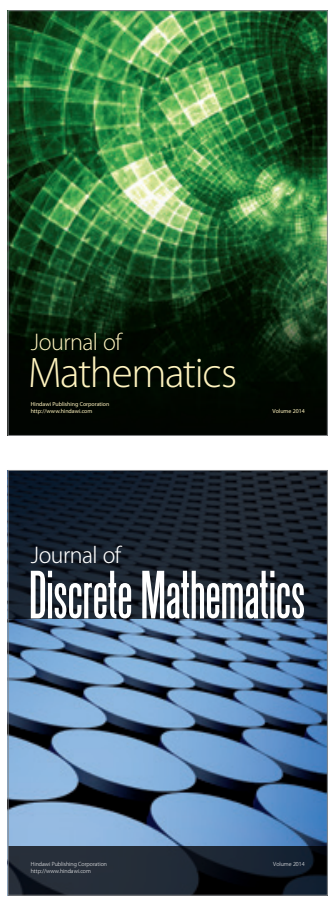

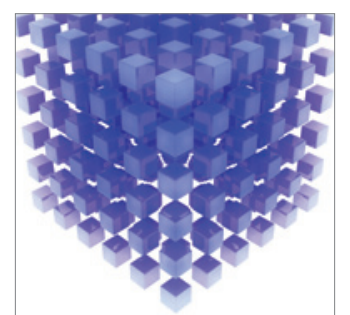

Mathematical Problems in Engineering
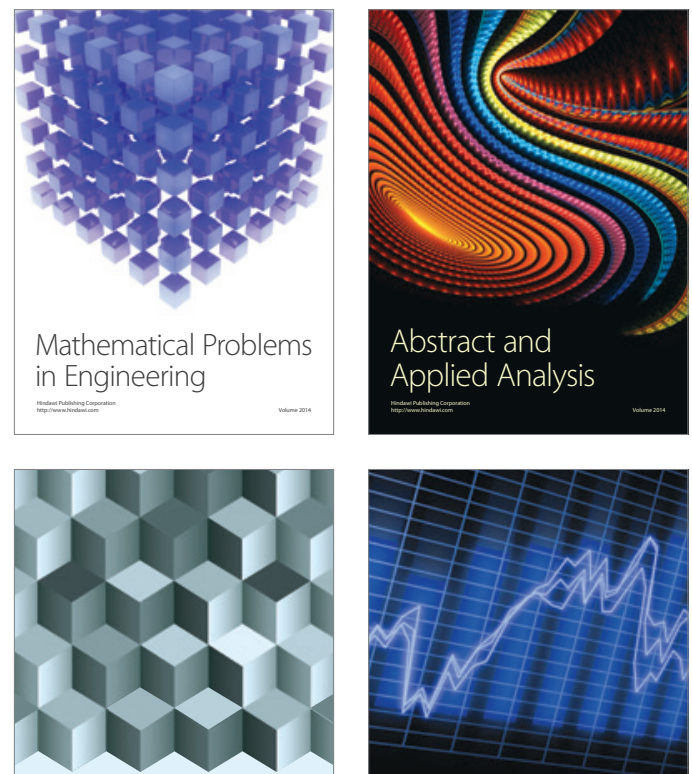

Journal of

Function Spaces

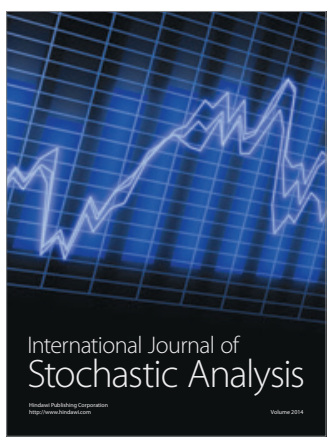

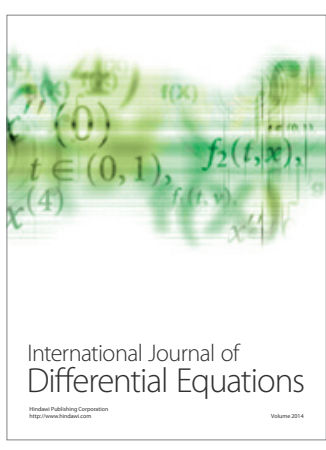
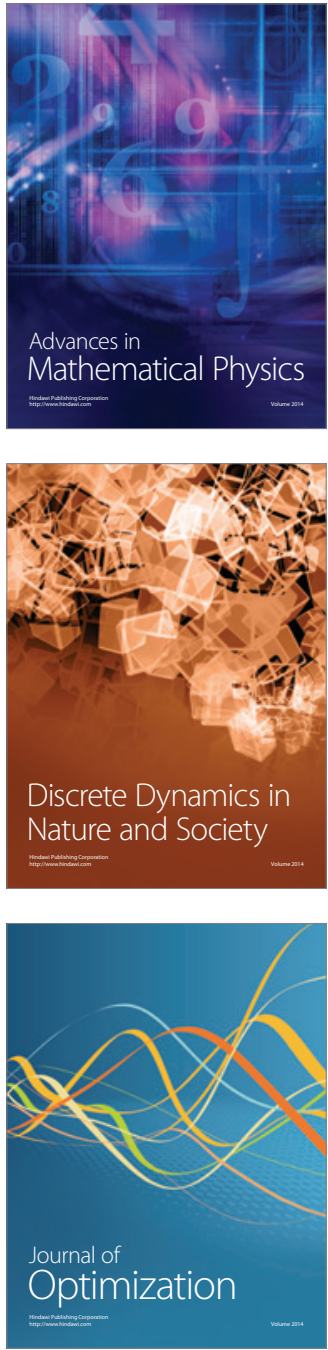